\title{
Randomised controlled trial of tyrosine supplementation on neuropsychological performance in phenylketonuria
}

Mary Lou Smith, William B Hanley, Joe T R Clarke, Paula Klim, Wanda Schoonheyt, Valerie Austin, Denis C Lehotay

\begin{abstract}
Objective-To test the efficacy of tyrosine supplementation, as an adjunct to dietary treatment, on neuropsychological test performance in individuals with phenylketonuria.

Design-A randomised controlled trial of tyrosine supplementation using a double blind crossover procedure with three four week phases.

Setting-The Hospital for Sick Children, Toronto.

Participants-21 individuals with phenylketonuria (ages 6 to 28 years, mean 11.3). Intervention-Participants were given 100 $\mathrm{mg} / \mathrm{kg}$ body weight/d of L-tyrosine or L-alanine (placebo).

Results-At baseline, performance on several of the neuropsychological test measures was correlated with tyrosine levels. Dietary supplements of tyrosine increased plasma tyrosine concentrations; however, no change in test performance was found across the tyrosine and placebo phases of the study.

Conclusions-Tyrosine supplementation of this type does not appear to alter neuropsychological performance in individuals with phenylketonuria.

(Arch Dis Child 1998;78:116-121)
\end{abstract}

University of Toronto and Hospital for Sick Children, Toronto, Canada:

Department of

Psychology

M L Smith

P Klim

Department of Pediatrics and Division of Clinical

Genetics

W B Hanley

J T R Clarke

W Schoonheyt

V Austin

Clinical Biochemistry D C Lehotay

Correspondence to: Dr Mary Lou Smith, Department of Psychology, Erindale College, University of Toronto, Mississauga, Ontario L5L 1C6, Canada. e-mail:

smithml@psych.utoronto.ca

Accepted 4 September 1997
Keywords: phenylketonuria; tyrosine; phenylalanine; frontal lobe function

When dietary phenylalanine restriction is instituted early in infancy, individuals with phenylketonuria develop with normal intelligence. ${ }^{1-3}$ None the less, they have been found to have specific behavioural and cognitive deficits that persist into adulthood, and discontinuation of treatment may result in deterioration in neuropsychological performance. ${ }^{4-15}$ At least part of these deficits may be reversible by changes in dietary phenylalanine levels. Using crossover designs with periods of high and low phenylalanine intake, Krause et $a l^{8}$ and Clarke et $a l^{6}$ explored the short term neurotoxic effects of hyperphenylalaninaemia on performance on choice reaction time tasks, showing a negative correlation between plasma phenylalanine level and reaction time. Furthermore, Clarke et al found a highly significant improvement in reaction time during the low phenylalanine phase.

Taken together, these observations suggest that at least some component of the neurotox- icity of hyperphenylalaninaemia is reversible by dietary phenylalanine restriction. The mechanism of this late, apparently reversible neurotoxicity is not known; however, it may involve neurotransmitter metabolism resulting from subtle unrecognised nutritional deficiencies. The enzymatic conversion of phenylalanine to tyrosine and the hydroxylation of tyrosine to form dihydroxyphenylalanine (dopa) are impaired by high phenylalanine concentrations. ${ }^{16}$ The transport of phenylalanine into brain is mediated by a carrier mechanism shared with other neutral amino acids, ${ }^{17} 18$ and phenylalanine inhibits their transport in vitro. ${ }^{19} 20$ Plasma phenylalanine concentrations commonly observed in patients with untreated phenylketonuria have been shown to impair the uptake by the brain of other neutral amino acids, including tyrosine and tryptophan, ${ }^{21}$ although more recent studies have shown that this effect is not as prominent as the effect on leucine transport into the brain..$^{22}$ High phenylalanine concentrations also result in sequestration of tyrosine in parenchymal tissue, such as liver. ${ }^{23}$

Tyrosine is a precursor of the neurotransmitters dopamine and noradrenaline, and tryptophan is the precursor of serotonin. In phenylketonuria, the concentrations of tyrosine and tryptophan in plasma and in brain are below normal. ${ }^{24}{ }^{25}$ Krause et al found that urinary dopamine excretion was inversely related to plasma phenylalanine in young adults with phenylketonuria. ${ }^{8}$ Lou et al reported that cerebrospinal fluid (CSF) concentrations of the dopamine and serotonin metabolites, HVA and 5-HIAA, respectively, were markedly decreased in phenylketonuric subjects when they became hyperphenylalaninaemic on unrestricted diets. ${ }^{26}$ Interestingly, despite marked increases in plasma phenylalanine concentrations, there was no consistent change in CSF tyrosine or tryptophan in their subjects.

Bessman first suggested that low brain tyrosine might be as critical as high phenylalanine in the damage caused to phenylketonuric patients. ${ }^{27}$ Studies testing that hypothesis have yielded mixed results. Lou et al showed increased neurotransmitter biosynthesis in two patients with phenylketonuria on supplementation with large doses of tryptophan (100 $\mathrm{mg} / \mathrm{kg}$ ) and tyrosine $(200 \mathrm{mg} / \mathrm{kg})$, and in addition found improved reaction times in the one patient who had abnormal vigilance. ${ }^{26}$ Lou et $a l^{28}$ and Lykkelund $e t a l^{29}$ studied patients with phenylketonuria who were allowed unre- 
stricted diets and underwent neuropsychological testing before and after tyrosine supplementation (in doses of 106 to $194 \mathrm{mg} / \mathrm{kg} /$ day). The addition of tyrosine resulted in an increased HVA/HIAA ratio in the CSF. Those patients who had abnormally long reaction times showed a decrease in the variability of these slow responses. Furthermore, an inverse relation between the dose of tyrosine and reaction time variability was reported in one patient supplemented with doses of tyrosine of up to $160 \mathrm{mg} / \mathrm{kg} /$ day.

Despite these encouraging results, more recent investigations have not been able to replicate the effects. Mazzocco et al found no improvement in cognitive performance in children on dietary treatment supplemented with $2500 \mathrm{mg} /$ day of tyrosine. Reaction time was not measured in their study. ${ }^{30}$ Pietz et al investigated supplementation with $100 \mathrm{mg} / \mathrm{kg} /$ day in patients who had relaxed or stopped the dietary regimen, ${ }^{31}$ and found no change in visual evoked potentials, EEG, or reaction time. However, the studies differed from those reported by Lou and others with respect to a number of variables, including the age of the patients studied (adults $v$ children), the measures used (reaction time tasks $v$ other measures of cognitive performance), the dose of tyrosine used, and the dietary status of the subjects.

Although the evidence for the efficacy of tyrosine supplementation in phenylketonuria is far from conclusive, many centres are using it as adjunctive treatment. Our present study was designed to test the effect of tyrosine supplementation as a treatment for phenylketonuria, and in so doing, to address the variables that differed across the previous studies. Subjects of a wide age range were included, and measures encompassed not only reaction time but also other aspects of cognitive performance. The dose $(100 \mathrm{mg} / \mathrm{kg} /$ day $)$ was chosen as approximately midway between that used by Lou $e t a l^{28}$ and by Mazzocco et $a l^{30}$ and was the same as to that of Pietz et al. ${ }^{31}$ Although this dose was not effective in Pietz's study, the subjects were on relaxed or unrestricted diets. We sought to determine whether this level of supplementation would be effective in subjects on a phenylalanine restricted diet. The supplementation phase was four weeks in length, which was considered to be adequate to demonstrate possible effects, given that Lou et al had shown changes after only three days of tyrosine supplementation. ${ }^{28}$

In choosing the test measures, we were influenced by recent work suggesting that the nature of the neuropsychological deficits in phenylketonuria is specific, and may involve dopamine pathways, particularly those in the frontal lobes. ${ }^{15}$ 32-34 Therefore, tests were selected to sample frontal lobe functions, and tasks thought not to tap the frontal lobes were used as control measures.

\section{Methods}

SUBJECTS

Twenty three individuals with phenylketonuria (12 females, 11 males) were recruited from the phenylketonuria clinic of the Hospital for Sick Children in Toronto. All subjects spoke English as a first language, were in good health, and were not taking any psychoactive drugs. Two subjects (both male) were not included in the final analyses: one admitted that he had not been taking his supplements consistently during the study, and one was not shifted from tyrosine to the placebo at a planned phase change, because of a pharmacy error.

The mean age of the final sample of 21 subjects was 11.3 years (SD 6.2; range 6 to 28), and their mean full scale intelligence quotient (IQ) (based on the age appropriate form of the Wechsler intelligence scale) was 102 (SD 11.2, range 82 to 123). All patients were on phenylalanine restricted diets, although in two cases the restriction was minimal. The intake of tyrosine in the amino acid supplements given as part of the dietary treatment was highly variable, with a maximum of $100-150 \mathrm{mg} / \mathrm{kg}$ body weight. At baseline, the mean plasma phenylalanine concentration was $785.3 \mu \mathrm{mol} / 1$ (SD 202.7, range 472 to $1148 \mu \mathrm{mol} / \mathrm{l}$ ), and the mean plasma tyrosine concentration was 41.6 $\mu \mathrm{mol} / 1$ (SD 12.1 ; range 24 to $67 \mu \mathrm{mol} / \mathrm{l})$.

\section{MATERIALS AND PROCEDURE}

The following tasks were administered in the baseline and three supplementation phases: two tests of choice reaction time (simple and vigilance reaction time tasks), a test of response organisation and monitoring (self ordered pointing task), two tests of response inhibition (delay task and conflicting motor task), and a task requiring short term memory with or without distraction. Choice reaction time tasks have been shown to be sensitive to dietary phenylalanine, ${ }^{6}$ to dietary levels of tyrosine ${ }^{28}$ to CSF HVA and 5-HIAA levels, ${ }^{28}$ and to plasma phenylalanine concentration. ${ }^{8}$ The remaining tasks tap abilities that have been shown to be sensitive to the effects of frontal lobe damage in adults, and have also been used in developmental studies on frontal lobe functioning. ${ }^{35-43} \mathrm{~A}$ word list memory $\operatorname{task}^{44}$ and a measure of receptive vocabulary ${ }^{45}$ were included as control tasks; alternate forms of these two tests were administered at baseline and at the end of the second supplementation phases only. A detailed description of the tasks is presented in the appendix.

All aspects of the study were conducted on an outpatient basis. A double blind crossover within subject study design was employed. All subjects were maintained on therapeutic low phenylalanine diets throughout the study. The dietary supplements consisted of an "anonymous" powder, which was either L-tyrosine (100 mg/kg/day) or the placebo, L-alanine (100 $\mathrm{mg} / \mathrm{kg} /$ day). Alanine was selected as a placebo because it is relatively tasteless compared with other amino acids, and it is harmless, being rapidly oxidised or converted to glucose.

The daily dose of powder was divided into two portions, to be taken in the morning and the afternoon, mixed in food. Two randomisations of three phases were used, either TAT or ATA, where $T$ refers to the tyrosine supplementation phase and A to the alanine, or placebo, phase. Subjects underwent baseline neuropsychological and biochemical testing, 
Table 1 Mean (SD) phenylalanine and tyrosine concentrations during the tyrosine supplementation and non-supplementation ${ }^{\star}$ phases

\begin{tabular}{lll}
\hline & \multicolumn{2}{l}{ Supplementation status } \\
\cline { 2 - 3 } & Tyrosine & Non-supplementation $^{\star}$ \\
\hline Phenylalanine $(\mu \mathrm{mol} / \mathrm{l})$ & $806.3(280.8)$ & $780.65(174.4)$ \\
Tyrosine $(\mu \mathrm{mol} / \mathrm{l})$ & $55.0(24.3)$ & $42.3(7.8)$ \\
Phenylalanine:tyrosine ratio & $18.1(6.4)$ & $20.0(7.3)$ \\
\hline
\end{tabular}

${ }^{\star}$ Non-supplementation consists of the average of performance during baseline and L-alanine phases.

and were then randomly assigned to an initial A or $\mathrm{T}$ phase. The assignment of subjects to the initial phase, and the dispensing of the powders, was done by a research pharmacist. The tyrosine supplementation status of the subjects was concealed from all others (the subjects, their parents, the physicians caring for the subjects, and the neuropsychologist) until the end of the study.

Each phase lasted four weeks. Neuropsychological and biochemical testing was repeated at the end of each phase. After four weeks on the initial phase (A or $\mathrm{T}$ ), each subject was tested and changed to the opposite phase (A to $\mathrm{T}$ or $\mathrm{T}$ to A) for a further four week period; this pattern was repeated for the third phase. In total, each subject underwent testing four times (baseline and at the end of each of the three phases).

Plasma phenylalanine and tyrosine concentrations were monitored by quantitative amino acid analysis performed by automated ion exchange chromatography (Beckman Model 7300 amino acid analyser).

\section{Results}

At baseline, plasma phenylalanine was significantly correlated with the following dependent measures: total words recalled on the word list memory task $(r=-0.73, \mathrm{p}<0.01)$, number of words recalled in the no distraction version of the short term memory test $(r=-0.75$, $\mathrm{p}<0.05)$, number of errors on the self ordered pointing task $(r=0.51, \mathrm{p}<0.05)$, and reaction time in the vigilance task $(r=0.57, \mathrm{p}<0.05)$. Tyrosine concentrations at baseline were significantly correlated with number correct on the delay task $(r=0.56, \mathrm{p}<0.05)$, number of errors on the self ordered pointing task $(r=$ $-0.57, \mathrm{p}<0.01)$, and the number correct $(r=$ $0.50, \mathrm{p}<0.05)$ and reaction time $(r=-0.45$, $\mathrm{p}<0.05)$ on the vigilance reaction time task. The correlation between phenylalanine and tyrosine was not significant $(r=-0.39$, $\mathrm{p}>0.05)$.

The results for the supplementation phases were analysed in two ways. First, the results for the subjects randomised to the ATA design and those randomised to the TAT design were analysed separately. In the second set of analyses, to increase statistical power all subjects were pooled and the data were averaged across all tyrosine phases and all non-tyrosine (baseline and placebo) phases. In both analyses, paired $t$ tests were conducted to investigate any changes in the biochemical or neuropsychological variables over time as a function of the supplementation status of the subject. These analyses yielded the same outcome, and thus only the combined subject analyses are reported. Also, no differences were found between subjects undergoing the ATA regimen and those undergoing the TAT regimen.

Mean plasma phenylalanine and tyrosine concentrations across the tyrosine supplementation and non-supplementation phases of the study are reported in table 1 . There was no significant change in phenylalanine $\left(t_{(1,20)}=0.18\right.$, $p>0.05$ ), but tyrosine levels were significantly higher on tyrosine supplementation than on placebo $\left(t_{(1,20)}=2.76, \mathrm{p}<0.05\right)$. However, the phenylalanine to tyrosine ratio did not vary significantly with the supplementation status of the subjects $\left(t_{(1,20)}=1.51, \mathrm{p}>0.05\right)$.

Table 2 gives the means and standard deviations for the measures administered across all four phases of the study, and the $t$ values and probabilities associated with the analyses. This table also contains the confidence intervals for the mean differences between the tyrosine and non-supplementation conditions, together with the standard errors for those mean differences. No significant differences were detected in any of the dependent variables.

Because Lou et al found their effect of tyrosine supplementation on reaction time variability, ${ }^{28}$ secondary analyses were con-

Table 2 Means (SD) across tyrosine supplementation and non-supplementation * phases, together with the confidence intervals and standard errors for the mean differences between the conditions

\begin{tabular}{|c|c|c|c|c|c|c|}
\hline Measure & Tyrosine & Non-supplementation * & $t(d f)$ & $p$ & $\begin{array}{l}\text { Confidence } \\
\text { interval }\end{array}$ & $S E$ \\
\hline \multicolumn{7}{|l|}{ Conflicting motor task } \\
\hline Number of errors & $5.07(2.72)$ & $5.69(2.47)$ & $-1.24(1,20)$ & 0.23 & -1.13 to -0.12 & 0.51 \\
\hline \multicolumn{7}{|l|}{ Delay task } \\
\hline Number correct & $50.31(13.53)$ & $46.60(12.67)$ & $1.76(1,17)$ & 0.10 & 1.59 to 5.81 & 2.11 \\
\hline Efficiency ratio & $0.81(0.16)$ & $0.80(0.17)$ & $0.42(1,17)$ & 0.68 & -0.02 to 0.05 & 0.04 \\
\hline \multicolumn{7}{|l|}{ Simple RT task } \\
\hline Reaction time (ms) & $624.0 \quad(119.7)$ & $619.5(139.0)$ & $0.42(1,18)$ & 0.68 & -6.17 to 15.11 & 10.64 \\
\hline Number correct $\max =30$ & $27.16(3.73)$ & $27.03(3.64)$ & $0.27(1,18)$ & 0.79 & -0.35 to 0.61 & 0.48 \\
\hline Errors of commission & $4.58(9.27)$ & $4.65(9.61)$ & $-0.05(1,18)$ & 0.96 & -1.44 to 1.30 & 1.37 \\
\hline \multicolumn{7}{|l|}{ Vigilance RT task } \\
\hline Reaction time (ms) & $454.3 \quad(96.6)$ & $469.9 \quad(99.0)$ & $-1.33(1,20)$ & 0.20 & -27.35 to -3.93 & 11.70 \\
\hline Number correct $\max =45$ & $39.45(6.25)$ & $39.96(4.55)$ & $-0.76(1,20)$ & 0.46 & -1.18 to 0.16 & 0.67 \\
\hline Errors of commission & $6.74(8.77)$ & $7.65(8.53)$ & $-0.83(1,20)$ & 0.42 & -2.01 to 0.19 & 1.10 \\
\hline \multicolumn{7}{|l|}{ Self ordered pointing task } \\
\hline Number of errors & $12.55(6.72)$ & $12.16(6.52)$ & $0.37(1,20)$ & 0.72 & -0.66 to 1.44 & 1.05 \\
\hline \multicolumn{7}{|l|}{ Short term memory task } \\
\hline Distraction condition & $35.50(11.85)$ & $35.52(11.85)$ & $1.27(1,7)$ & 0.25 & 0.63 to 5.32 & 2.35 \\
\hline No distraction condition & $76.56(3.33)$ & $76.88(4.68)$ & $-0.23(1,7)$ & 0.83 & -1.69 to 1.07 & 1.38 \\
\hline
\end{tabular}

^Non-supplementation consists of the average of performance during baseline and L-alanine phases.

$\mathrm{df}=$ degrees of freedom; $\mathrm{RT}=$ reaction time. 
ducted on the reaction time data to evaluate variability in responding during each task. Each reaction time task was divided into three blocks, and the largest difference in mean reaction time across the three blocks was recorded for each subject. These difference scores were subjected to repeated measures of analyses of variance to examine for effects related to supplementation status. The analyses revealed no differences in variability between the tyrosine and placebo phases for either the simple task $\left(t_{(1,18)}=0.29, \mathrm{p}>0.05\right)$ or the vigilance $\operatorname{task}\left(t_{(1,18)}=0.39, \mathrm{p}>0.05\right)$.

Two tasks (receptive vocabulary and word list memory) were administered on only two occasions, in the baseline phase and at the end of the second supplementation phase. For subjects in the ATA randomisation, the second administration of these tasks occurred during a tyrosine phase, and for the subjects in the TAT randomisation, the second administration corresponded to a placebo phase. Performance on these two tasks was analysed in terms of score change: the difference between performance at baseline and at the second administration. For receptive vocabulary, there was no significant difference $\left(t_{(1,19)}=1.15, \mathrm{p}>0.05\right)$ in the change scores seen with tyrosine supplementation (change $=-1.62$, SD 8.5) and with the placebo (change $=2.75, \mathrm{SD} 8.3$ ). Similar results were obtained for the word list memory task, where again the change scores did not differ significantly with supplementation status $\left(t_{(1,19)}=\right.$ $1.15, \mathrm{p}>0.05)$; the score change for the subjects on tyrosine was 3.23 (SD 5.5), and for subjects on the placebo it was 0.50 (SD 4.9).

\section{Discussion}

Although no effect of tyrosine supplementation on neuropsychological test performance was found, the findings provide further evidence for a long term biochemical basis for the neuropsychological deficits associated with early treated phenylketonuria. Significant correlations between concurrent phenylalanine levels and task performance were obtained for several of the neuropsychological measures. Higher phenylalanine levels were associated with poorer performance on tests of verbal memory, response organisation, and reaction time under conditions of sustained vigilance. These results add to the solid body of findings indicating the neurotoxic effects of phenylalanine. ${ }^{46814152832}$

Indices of response organisation, reaction time, stimulus monitoring, and impulsive responding were also correlated with plasma levels of tyrosine. These latter findings extend previous work showing a relation between reaction time variability and plasma tyrosine in patients with phenylketonuria. ${ }^{28}{ }^{29}$ These results could be interpreted as either confirming the important role of tyrosine in the chain of neurochemical events that underlie cognitive performance in patients with phenylketonuria, or as simply a general reflection of dietary control. The latter, simpler explanation may not be sufficient to explain the association between better test performance and higher tyrosine levels. First, tyrosine and phenylalanine levels were not significantly correlated, as one might expect if dietary control totally explained the findings. Second, the neuropsychological variables which correlated with the plasma biochemical variables differed for tyrosine and phenylalanine. Again, one would assume that better dietary control would lead to lower phenylalanine and higher tyrosine and thus influence the same tasks. It is therefore possible that tyrosine may be exerting an independent effect. This possibility is supported by previous findings in patients with atypical phenylketonuria or mild hyperphenylalaninaemia, in whom no correlations between phenylalanine and IQ measures were seen, but in whom tyrosine levels correlated significantly with the performance IQ, which comprises tasks of nonverbal reasoning and visuomotor functioning. ${ }^{47}$

In this study, supplementation with tyrosine in patients with phenylketonuria who were on low phenylalanine diets resulted in a significant increase in plasma tyrosine. None the less, this increase was not associated with any change in performance, relative to that seen with administration of a placebo, on reaction time or cognitive tasks. Lou et al had originally shown an effect of tyrosine supplementation on reaction time variability ${ }^{28}$; we were unable to replicate the effect with our measure of reaction time variability or with any of the other tasks. Thus our results support the conclusion arising from other recent studies, that tyrosine supplementation appears to be of no benefit to individuals with phenylketonuria, whether or not they are restricting dietary intake of phenylalanine. This conclusion appears to apply not only to performance on neuropsychological measures, such as demonstrated here and by others, ${ }^{30} 31$ but also to neurophysiological indices of brain function, including visual evoked potentials and EEG. ${ }^{31}$ Because of recent evidence indicating that patients with phenylketonuria have cognitive deficits related to frontal lobe abnormality, ${ }^{15} 3234$ the test battery in the present study had used measures sensitive to frontal lobe functioning, suggesting that the negative results were not due to inappropriate task selection.

In the present study, the dietary supplements of tyrosine resulted in an average increase in plasma tyrosine concentrations such that they were $130 \%$ of the baseline value. This increase is smaller than that reported in other studies. Pietz et al found tyrosine levels of $200 \%$ of baseline values with the same dose, ${ }^{31}$ and Lou reported a mean increase to $276 \%$ of baseline with his higher doses of tyrosine (106-194 $\mathrm{mg} / \mathrm{kg} /$ day). ${ }^{29}$ In normal volunteers, a value of $223 \%$ of baseline plasma tyrosine concentrations has been demonstrated two to eight hours after ingesting $100 \mathrm{mg} / \mathrm{kg}$ of tyrosine in a single dose. ${ }^{46}$ As Pietz et al have suggested, these differences across studies may be due to the differences in administration, as for example in capsule alone or powder mixed with food, or ingested at one time as opposed to spread out during the day.

In understanding the consistent failure to replicate the results reported by Lou and colleagues, ${ }^{28}$ considerations of dosage may be important. The tyrosine supplement of 200 $\mathrm{mg} / \mathrm{kg}$ employed by them was at least twice as 
high as that used in all subsequent investigations. It may be that the positive results obtained were due to greater availability of tyrosine in the brain to compete with phenylalanine for transport across the blood-brain barrier. This possibility has not been addressed directly in any of the studies reported, and would be difficult to assess in an ethical manner in human subjects.

What is not known in the present study or other recent investigations is the effect of dietary supplementation on brain levels of tyrosine. Of more importance than the plasma tyrosine level itself is the ratio of phenylalanine to tyrosine, since phenylalanine competes with tyrosine for carrier proteins at the blood-brain barrier. Although the dietary supplements increased the amount of tyrosine in plasma, they did not significantly alter the phenylalanine to tyrosine ratio, which was approximately 18:1 when subjects were ingesting tyrosine and 20:1 when they were not. Both of these ratios were well above the normal $1: 1$ ratio.

Various potential reasons why we failed to find a supplementation effect were considered. One is that the subjects may not have been compliant with ingesting the dietary supplements. Detailed dietary records were kept during the study, and were reviewed by one of the authors (VA), a registered dietitian. With only one exception (whose data were not included in the study), all participants reported being compliant with the supplementation regimen. It is also possible that the clinical heterogeneity of the sample of subjects prevented the expression of treatment effects. There may be a genetic component to endogenous tyrosine levels, or it may be that tyrosine concentrations early in life may influence cognitive development, and there is a critical (early) age after which cognitive development cannot be altered by dietary supplementation, at least at the levels of tyrosine employed in this study. Finally, the four week supplementation period may have been insufficient for possible effects to be seen; this possibility is unlikely, given that Lou et al found an effect after only three days of tyrosine supplementation. ${ }^{28}$

Many centres that follow patients with phenylketonuria have adopted tyrosine supplementation as an adjunct method of treatment. The baseline correlations between tyrosine and task performance suggest that tyrosine availability may play an important part in the neuropsychological performance of patients with phenylketonuria. However, our results show that, although dietary tyrosine supplements increased plasma concentrations of tyrosine, they did not have an effect on neuropsychological test performance under the conditions of our study. The supplementation we employed would not appear to be successful in offsetting the cognitive deficits in phenylketonuria. On the other hand, the findings suggest that further studies are necessary to determine whether alternate methods of administration may be more effective in increasing the availability of tyrosine to the brain. This research was funded by grants from the Ontario Mental
Health Foundation and the Velleman Foundation. We are gratth Foundation and the Velleman Foundation. We are grateful to all the participants and their families for the time and
enthusiasm they dedicated to this project, to Michael Wasdell enthusiasm they dedicated to this project, to Michael Wasdell
for assistance with the statistical analyses, to Jennifer Saltzman for assistance with the statistical analyses, to Jennifer Saltzman
for editorial assistance, and to anonymous reviewers for their suggestions for improvements to the manuscript.

\section{Appendix}

DESCRIPTION OF TASKS

Two reaction time tasks, taken from the Gordon diagnostic system, ${ }^{38}$ a self contained, electronic testing device, were administered. In the first task, named the simple task, subjects viewed a random series of numbers from 0 to 9 , which appeared for $200 \mathrm{~ms}$ every $2 \mathrm{~s}$ in the centre of a three column electronic display; subjects were required to respond as quickly as possible by pressing a button whenever a 0 appeared. There was a total of 30 targets. In the vigilance task, based on the continuous performance test, ${ }^{39}$ they had to respond only when a 9 appeared which had been preceded by a 1 . The digits each appeared for $200 \mathrm{~ms}$ at a presentation rate of 1 per second. This task contained 45 targets. Reaction time, number correct, and errors of commission were recorded for each task.

The self ordered pointing task, adapted from Petrides and Milner, ${ }^{40}$ requires response organisation, planning, attention, and short term memory. Subjects were presented with stacks of $21.5 \times 28 \mathrm{~cm}$ pages, each containing a matrix of $6,8,10$, or 12 pictures of familiar objects. No picture appeared in more than one matrix set. Within a matrix set, the same pictures appeared on each page, but their relative positions on the page varied randomly. Subjects were instructed to touch each picture in the set, by going through the pages one at a time, and touching one picture on each page. Pictures could be touched in any order, but each picture was to be touched only once. Subjects were told not to point to the same spatial location on consecutive pages. Three trials were given for each set of pictures, beginning with the six item set and progressing to the 12 item set. Number of errors was summed across all trials for all sets.

Two measures of response inhibition were used: the delay task and the conflicting motor task. The delay task, also from the Gordon diagnostic system, ${ }^{38}$ measures the ability to suppress or delay impulsive behavioural response. The subject was instructed to press a button, wait an unspecified time, and then press the button again. If the subject refrained from responding for at least $6 \mathrm{~s}$, a light flashed and a reward counter incremented. If the subject responded before the interval elapsed, the timer reset and no reward points were obtained. The task yielded two scores: total number of responses and the efficiency ratio, which represents the percentage of correct responses from the total responses.

The conflicting motor response task, adapted from Christensen, ${ }^{41}$ required the subject to perform motor actions opposite to that modelled by the examiner. Subjects were trained to make two gestural signals ("Show me your fist," "Show me your finger"). They were then told that when the examiner showed a fist, they were to show a finger, and that when the exam- 
iner showed a finger, they were to make a fist. Each of these two gestures was presented 40 times in a predetermined random sequence at a rate of one gesture per second. Number of errors was recorded.

The short term memory task, modelled after work by Brown ${ }^{42}$ and Peterson and Peterson, ${ }^{43}$ was administered only to subjects 11 years and older. There were two versions of the task, each containing 10 trials of four words which subjects were asked to recall, in the order in which they were presented, after a delay of 20 seconds. In the no distracter version of the task, this delay was left unfilled, whereas in the distracter version, the subject was given a three digit number and was asked to count backwards from that number until the interval had elapsed. The order of presentation of these two versions was counterbalanced. A maximum of eight points per trial was awarded, one point for each word recalled, and one point for the order in which the word was recalled.

In the word list memory task, subjects were read 12 unrelated words (taken from the lists used by Hannay and Levin, ${ }^{44}$ over three repeated trials. Recall was tested following each trial, and after a delay of 15 minutes. The dependent variable was the sum of words recalled across the three trials and the delay trial.

In the Peabody Picture Vocabulary Test-Revised, ${ }^{45}$ a measure of receptive vocabulary, the subject was shown a series of pictures, four to a page. For each item, the subject was asked to choose the picture that corresponded to a word spoken by the examiner. Number of correct items was converted to a standard score (mean of 100, SD 15) based on norms for the subject's age.

1 Berry HK, Bofinger MK, O'Grady DJ, Perlmutter LJ. Intellectual development and academic achievement of children treated early for phenylketonuria. Dev Med Child Neurol treated early for

2 Koch R, Azen C, Friedman EG, Williamson ML. Paired comparison between early treated phenylketonuria children and their matched sibling controls on intellectual and
school achievement test results at eight years. $\mathcal{F}$ Inherit school achievement test

3 Smith I, Beasley MG, Ades AE. Intelligence and quality of dietary treatment in phenylketonuria. Arch Dis Child 1990 65:472-8.

4 Brunner RL, Jordan MK, Berry HK. Early-treated phenylketonuria: neuropsychologic consequences. F Pediat 1983;102:831-5.

5 Cabalska B, Duszynska N, Borzymowska J, Zorska K, Koslacz-Folga A, Bozkowa K. Termination of dietary treatment in phenylketonuria. Eur F Pediatr 1977;126:253-62.

6 Clarke JTR, Gates RD, Hogan SE, Barrett M, MacDonald GW. Neuropsychological studies on adolescents with phenylketonuria returned to phenylalanine-restricted diets. Am f Ment Retard 1987;92: 255-62.

7 Crowie VA Neurological and psychiatric aspects of phenylketonuria. In: Bickel $\mathrm{H}$, Hudson $\mathrm{F}$, Woolf $\mathrm{L}$, eds. Phenylketonuria and some inborn errors of amino acid metaboPhenylketonuria and some inborn

8 Krause W, Halminski M, McDonald L, et al. Biochemical and neuropsychological effects of elevated plasma phenylalanine in patients with treated phenylketonuria. F Clin Invest 1985;75:40-8

9 Pennington BF, Van Doorninck WJ, McCabe LL, McCabe ERB. Neuropsychological deficits in early treated phenylketonuric children. Am f Ment Def 1985;89:467-74.

10 Ris MR, Williams SE, Hunt MM, Berry HK, Leslie N Early-treated phenylketonuria: adult neuropsychologic outcome. F Pediatr 1994;124:388-92.

11 Seashore MR, Friedman E, Novelly RA, Bapat V. Loss of intellectual function in children with phenylketonuria after relaxation of dietary phenylalanine restriction. Pediatrics 1985;75:226-32.

12 Smith I, Beasley MG, Wolff OH, Ades AE. Behavior disturbance in eight year old children with phenylketonuria. $\mathcal{f}$ Pediatr 1988;112:403-8

13 Smith I, Beasley MG. Intelligence and behavior in children with early treated phenylketonuria. Eur $\mathcal{F}$ Clin Nutr 1989;S43:1-5.
14 Smith ML, Klim P, Mallozzi E, Hanley WB. A test of the frontal specificity hypothesis in the cognitive performance of adults with Phenylketonuria. Dev Neuropsychol 1996;12: $327-41$

15 Welsh MC, Pennington BF, Ozonoff S, Rouse B, McCabe ERB. Neuropsychology of early-treated phenylketonuria: specific executive function deficits. Child Dev 1990;61: 1697-713.

16 Kaufam S. The phenylalanine hydroxylating system from mammalian liver. Adv Enzymol 1969;35:245-319.

17 Murray JE. Carrier-mediated transfer of amino acids from blood to brain. Neurology 1973;23:940-4.

18 Snodgrass SR, Cutler RWP, Kang ES, Lorenzo AV. Transport of neutral amino acids from feline cerebrospinal fluid. Am f Physiol 1969;217:974-80.

19 Guroff G, King W, Udenfriend S. The uptake of tyrosine by rat brain in vitro. $\mathcal{F}$ Biol Chem 1961;236:1773-7.

20 Neame KD. Phenylalanine as inhibitor of transport of amino acids in brain. Nature 1961;192:173-4.

21 Oldendorf WH. Saturation of blood brain barrier transport of amino acids in phenylketonuria. Arch Neurol 1973;28:45-8.

22 Knudsen GM, Hasselbalch S, Toft PB, Christensen E, Paulson OB, Lou H. Blood-brain barrier transport of amino acids in healthy controls and in patients with phenylketonuria. F Inher Metab Dis 1995;18:653-64.

23 Christensen HN. Where do the depleted amino acids go in phenylketonuria? Biochem f 1986;326:929-30.

24 Nord AM, McCabe L, McCabe ERB. Biochemical and nutritional status of children with hyperphenylalaninaemia. F Inher Metab Dis 1988;11:431-2.

25 McKean CM. The effect of high phenylalanine concentration on serotonin and catecholamine metabolism in the human brain. Brain Res 1972;47:469-76.

26 Lou HC, Guttler F, Lykkelund C, Bruhn P, Niederwieser A. Decreased vigilance and neurotransmitter synthesis after discontinuation of dietary treatment for phenylketonuria in adolescents. Eur 7 Pediatr 1985;144:17-20.

27 Bessman SP. The justification theory: the essential nature of the non-essential amino acids. Nutr Rev 1979;37:209-20.

28 Lou HC, Lykkelund C, Gerdes AM, Udesen H, Bruhn P. Increased vigilance and dopamine synthesis by large doses of tyrosine or phenylalanine restriction in phenylketonuria. Acta Paediatr Scand 1987;76:560-5

29 Lykkelund C, Nielson JB, Lou HC, et al. Increased neurotransmitter biosynthesis in phenylketonuria induced by phenylalanine restriction or by supplementation of unrestricted diet with large amounts of tyrosine. Eur F Pediatr 1989;148:239-45.

30 Mazzocco MMM, Yannicelli S, Nord AM, Van Doorninck W, Davidson-Mundt AJ, Greene CL. Cognition and tyrosine supplementation among school-aged children with phenylketonuria. Am F Dis Child 1992;146:1261-4.

31 Pietz J, Landwehr R, Kutscha A, Schmidt H, de Sonneville Pietz J, Landwehr R, Kutscha A, Schmidt H, de Sonneville
L, Trefz FK. Effect of high-dose tyrosine supplementation on brain function in adults with phenylketonuria. F Pediatr 1995;127:936-43.

32 Diamond A. Phenylalanine levels of $6-10 \mathrm{mg} / \mathrm{dl}$ may not be as benign as once thought. Acta Pediatr Scand suppl 1994;407:89-91.

33 Diamond A, Ciaramitaro V, Donner E, Dhali S, Robinson MB. An animal model of early-treated phenylketonuria. $\mathcal{F}$ Neurosci 1994;14:3072-82.

34 Weglage J, Pietsch M, Funders B, Koch HG, Ullrich K. Deficits in selective and sustained attention processes in early treated children with phenylketonuriatr 1996;155. $200-4$.

35 Becker MG, Isaac W, Hynd GW. Neuropsychological development of non-verbal behaviors attributed to "frontal opment of non-verbal behaviors attributed to "f

36 Passler MA, Isaac W, Hynd GW. Neuropsychological development of behavior attributed to frontal lobe functioning in children. Dev Neuropsychol 1985;1:349-70.

37 Shue KL, Douglas VI. Attention deficit hyperactivity disorder and the frontal lobe syndrome. Brain Cogn 1992;20 $104-24$

38 Gordon M, Mettelman B. The assessment of attention. I. Standardization and reliability of a behavior-based measure. F Clin Psychol 1988;44:682-90.

39 Rosvold H, Mirsky A, Sarason I, Beck A. A continuous performance test of brain damage. F Consult Psychol 1956;20: 343-52.

40 Petrides M, Milner B. Deficits on subject ordered tasks after frontal- and temporal-lobe lesions in man. Neuropsychologia 1982;20:249-62.

41 Christensen AL. Luria's neuropsychological investigation. New York: Spectrum, 1975.

42 Brown J. Some tests of the decay theory of immediate memory. $Q$ F Exp Psychol 1958;10:12-21.

3 Peterson LR, Peterson MJ. Short-term retention of individual verbal items. F Exp Psychol 1959;58:193-8.

44 Hannay JH, Levin HS. Selective reminding test: an examination of the equivalence of four forms. F Clin Exp Neuropsychol 1985;7:251-63.

45 Dunn LM, Dunn LM. Peabody picture vocabulary test-revised manual. Circle Pines, MN: American Guidance Service, 1981.

46 Glaeser BS, Melamed E, Growdon JH, Wurtman RJ. Elevation of plasma tyrosine after a single dose of L-tyrosine. Life Sci 1979;25:265-71.

47 Waisbren SE, Schnell R, Levy HL. Intelligence and personality characteristics in adults with untreated atypical phenylketonuria and mild hyperphenylalaninemia. F Pediatr 1984;105:955-8. 